\title{
City Sizes May Affect Blackout Probabilities
}

\author{
The probabilities of electricity blackouts may be influenced by the sizes of \\ cities more than by the details of power grids.
}

By Philip Ball

E lectric power blackouts can occur on all scales, from local outages to country-wide failures. The probability of a given event depends on the size of the region it affects, according to a mathematical relationship called a power law. The reason for the power law hasn't been clear, but a new model suggests that it results from the same kind of distribution in the sizes of cities [1]. The model's creators say that understanding the factors that influence blackout probabilities could help engineers make electricity grids more robust.

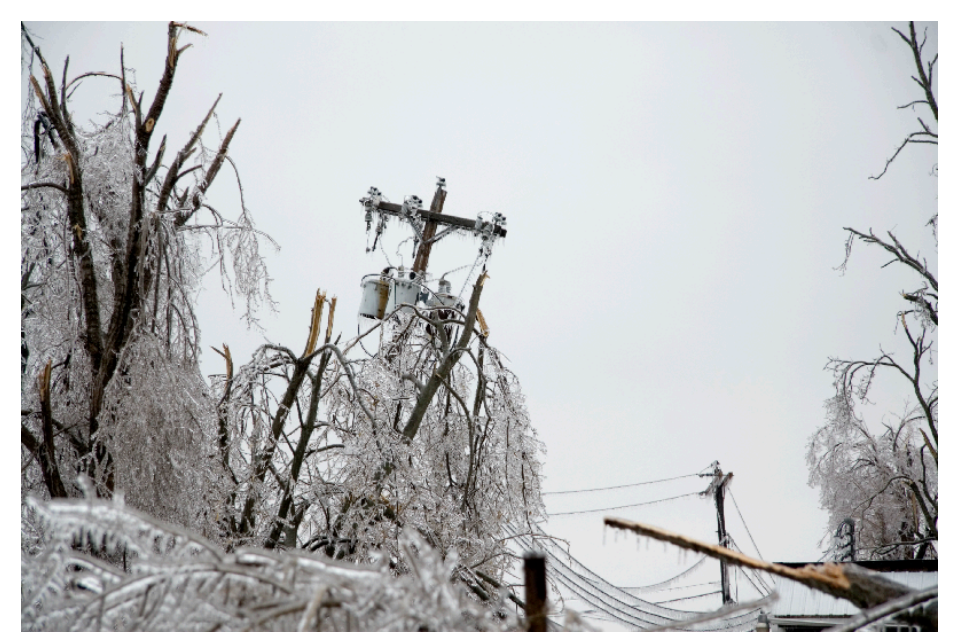

An avalanche of failure. Large-scale blackouts can be triggered by small events, such as the failure of a power line like this one in Missouri in 2007, caused by an ice storm. A new model suggests that the explanation for the probabilities of various blackout sizes lies not in the internal dynamics of blackout events but in the distribution of city sizes.

Credit: Getty Images/Bryngelzon
Massive power failures pose risks to public health, data storage, and social stability. Typically, blackouts happen when some failure such as the severing of a power line diverts power to neighboring parts of the grid and overloads them. A cascade of failures may then spread through the network like an avalanche. The power-law statistics mean that very large events, while more rare than small ones, occur much more often than would be expected if blackouts were governed by the normal or "Gaussian" statistics of random, independent events. A distribution with this feature is said to be "heavy-tailed."

In one popular class of models, the analogy with avalanches is literal: the cascade resembles the way a few tumbling rocks can collide with others to trigger a landslide, in a process described by a theory called self-organized criticality [2]. But such models don't take into account the physical processes that produce breakdowns in electricity grids.

Mathematician Bert Zwart of the Dutch National Research Institute for Mathematics and Computer Science (CWI) and his colleagues have now proposed another explanation. They point out that city sizes in any region tend also to have a power-law size distribution, and they say that this distribution might create the same statistics for electricity blackouts.

That's not as obvious as it might sound. If cities produced all their own power locally, their heavy-tailed size distribution wouldn't matter; outages would stay localized. Failure only spreads if a significant fraction of a city's power is actually generated elsewhere, says Zwart.

The researchers model the electricity grid as a real electrical 
circuit, with nodes representing cities with power consumption proportional to their populations and with links representing wires with fixed power-flow capacities. When a random failure of a specific line redistributes the flow, other lines may then exceed their limits and also fail. This cascade continues until no remaining lines exceed their limits. The blackouts, the researchers say, are then a result of a mismatch between the supply-the capacity of the links feeding into a given node-and the demand for power by the node.

The team's mathematical analysis and computer simulations of the model applied to idealized urban networks with power-law city size distributions yield failure statistics that also follow a power law. However, with a non-power-law distribution of city sizes, the model does not produce a heavy-tailed distribution of blackouts.

The researchers also looked at the types of power outages the model predicts for a real electricity grid, the one serving Germany. Zwart says that, although the German network is too small to see full power-law behavior (less than 600 nodes), the vast majority of large-scale blackouts in the German grid simulations came from the failure at a single node (a large city being cut off, say). In other words, even a small triggering event could sometimes have a big effect-a characteristic feature of heavy-tailed distributions. The characteristic mode of failure that results in power laws was thus still visible, Zwart says.
Marc Timme, a specialist in network dynamics at the Technical University of Dresden, Germany, says that this analysis "may substantially revise the way people reason about the observed power law." But he stresses that the model is a simplification. Marc Barthelemy, a physicist who studies urban networks at the French Alternative Energies and Atomic Energy Commission (CEA) in Saclay, echoes this concern. "It seems to me that the electrical network cannot reasonably be described by a network where the nodes are cities," he says-in a real grid there is more complexity, with a hierarchy of subsystems, from power plants to transformers and relays that transmit the power.

Zwart agrees that the model doesn't yet include the level of detail that might be needed to address complex engineering questions. But he hopes that the new approach might help in efforts to make power grids more robust against blackouts-for example, by helping researchers identify weak spots.

This research is published in Physical Review Letters.

Philip Ball is a freelance science writer in London. His latest book is How To Grow a Human (University of Chicago Press, 2019).

\section{REFERENCES}

1. T. Nesti et al., "Emergence of scale-free blackout sizes in power grids," Phys. Rev. Lett. 125, 058301 (2020).

2. B. A. Carreras et al., "Complex dynamics of blackouts in power transmission systems," Chaos Int. J. Nonlin. Sci. 14, 643 (2004). 